\title{
Accident Detection and Safety System - (AUTO AID APP)
} doi : https://doi.org/10.32628/CSEIT217413

\section{Suhaib Ahmed, Srinidhi N, Sandhya Kyamma, Mohammed Imran}

U.G Scholar, Department of Computer Science and Engineering, the National Institute of Engineering, Mysuru, Karnataka, India

\section{Article Info}

Volume 7, Issue 4

Page Number : 62-66

Publication Issue :

July-August-2021

\section{Article History}

Accepted : 02 July 2021

Published : 08 July 2021

\section{ABSTRACT}

Nowadays we are able to track vehicles using many applications which help in securing personal vehicles, public vehicles, feet units and others. Furthermore there is a rapid increase in the occurrence of the Road accident. This project is about a system which is developed to automatically detect an accident and alert the nearest hospitals and medical services about it. This system can also locate the place of the accident so that the medical services can be directed immediately towards it. The goal of this paper is to build up a Vehicle accidental monitoring system using MEMS, GPS and GSM Technology. The system comprises of accelerometer, MCU, GPS \& GSM Module support in sending message. The accelerometer is used to detect fall and Threshold Algorithm are used to detect accident. Short Message will contain GPS [Latitude, Longitude] which helps in locating the vehicles.

Keywords : Traffic accidents, accident detection, on-board sensor, MEMS Sensor, Vibration Sensor, accelerometer, android smart phones, real-time tracking, emergency services, emergency responder, emergency victim

\section{INTRODUCTION}

At present time in country like India where there is high population and due to urbanization there is a high demand of cars by the people which has eventually led to increase road traffic and accidents, thus keeps the lives of people at high risk. Street car crashes has driven for in excess of 1.25 million deaths worldwide each and every year and in excess of two hundred thousand deaths in India alone in 2013, as detailed by the World Health Organization [1]. Due to employment the usage of vehicles like cars, bikes can be increased, because of this reason the accidents can be happened due to over speed. People are going under risk because of their over speed, due to unavailability of advanced techniques, the rate of accidents can't be decreased. To reduce the accident rate in the country this paper introduces a optimum solution. Automatic alert system for vehicle accidents is introduced; the main objective is to control the accidents by sending a message to the registered mobile using wireless communications techniques. When an accident occurs at a city, the message is sent to the registered mobile through GSM module in less time. Arduino is the heart of the system which helps in transferring the message to different devices in the system. Vibration sensor will be activated when the accident occurs and the information is transferred to

Copyright: @ the author(s), publisher and licensee Technoscience Academy. This is an open-access article distributed under the terms of the Creative Commons Attribution Non-Commercial License, which permits unrestricted non-commercial use, distribution, and reproduction in any medium, provided the original work is properly cited 
the registered number through GSM module. GPS system will help in finding the location of the accident spot. The proposed system will check whether an accident has occurred and notifies to nearest medical centres and registered mobile numbers about the place of accident using GSM and GPS modules. The location can be sent through tracking system to cover the geographical coordinates over the area. The accident can be detected by a vibration sensor which is used as major module in the system.

In this paper we are using a alarm system which helps in improving the emergency system of the accident system. This system detects the accident occurrence and the co-ordinate of the accident are messaged to the rescue team .A switching system is used switch off in case there are no causality. The Accident is detected with the help of MEMS Sensor and Vibration Sensor. The Angle in which the car has rolled off is indicated through a message. This Application helps in providing feasible solution to the poor emergency facilitates.

\section{METHODS AND MATERIAL}

There are various working systems and research papers related to road safety and accident detection: Paper [1] Suggests that "The best strategy for a road safety approach is to ensure that in the event of a crash the energy of the impact on the concerned remains lesser than a threshold that might cause any significant permanent/non permanent damage." This is however not always possible and thus one must take into account the cases where damage is inflicted upon the user. The best bet in such scenarios is the providence of proper medical care.

Another research paper [2] suggests a way of accident detection using GPS and CCTV. This proposition however has various drawbacks, and video analysis for accident is quite complicated and may not detect few accidents. Also, the accidents that happen outside town bounds, where there is no CCTV, will go undetected. Good road quality and proper infrastructure also reduces the number of accidents and any other road mishaps in general. This approach, however, deals with the prevention of the accident and not with its aftermath.

While the principle of accident detection using accelerometer has existed since late twentieth century, it has now gained prominence as most smart phones nowadays have accelerometer built-in. The research paper [4] brings out importance of using accelerometer as primary component for accident. It suggests that the Accelerometer based transportation system could be helpful in sensing the change in gforces and also provide automation emergency calling system. This system may help to save the life of accident victim by providing information about accident detection or crash detection through GSM module. Paper [5] suggests an auto dialler system to provide address and other pertinent data to a central monitoring point or PSAP without caller intervention, that is, when activated by an external signal. In particular, it could provide a warning of a medical condition of the caller and automatically request, or indicate the need for, dispatch of an ambulance. The system also recognizes the need to insure the security of the transmitted information so that an outside party cannot access it with an incoming call.

\section{TECHNOLOGIES UTILIZED}

\section{A. Smart phones}

Smart phones are mobile phones that have considerably a lot of functionality than a regular mobile phone. They're mobile computers. Smart phones are powerful and versatile as a result of built in sensors, powerful processors, multiple network 
interfaces and a high amount of memory for such small devices.

B. Google Geolocation and Geocoding API:

Geocoding is the process of converting addresses (like "1600 Amphitheatre Parkway, Mountain View, CA") into geographic coordinates (like latitude 37.423021 and longitude - 122.083739), which you can use to place markers on a map, or position the map. Reverse geocoding is the process of converting geographic coordinates into a human-readable address. You can also use the Geocoding API to find the address for a given place ID.

\section{Accelerometer}

An accelerometer works by detecting proper acceleration affecting the accelerometer to determine the G-forces affecting the accelerometer [15]. Proper acceleration means acceleration that is relative to free-fall [16].

\section{Sending Notifications and SMS:}

Android provides a range of classes and interfaces to implement the SMS functionality hence eliminating the need for 3 rd party services to send sms and push notifications. The SmsManager android class enables us to send customized messages to the user's emergency contacts.

\section{SYSTEM IMPLEMENTATION}

The flow chart (a) shows the case where a moving vehicle hit another vehicle or static object and suddenly came to rest, whereas the flow chart (b) shows the case where a vehicle at rest was hit by another vehicle moving at high speed. The accident detection happens depending on the instantaneous acceleration value and time of contact and for ideal accident detection it is $+/-50 \mathrm{~m} / \mathrm{s}^{-2}$.
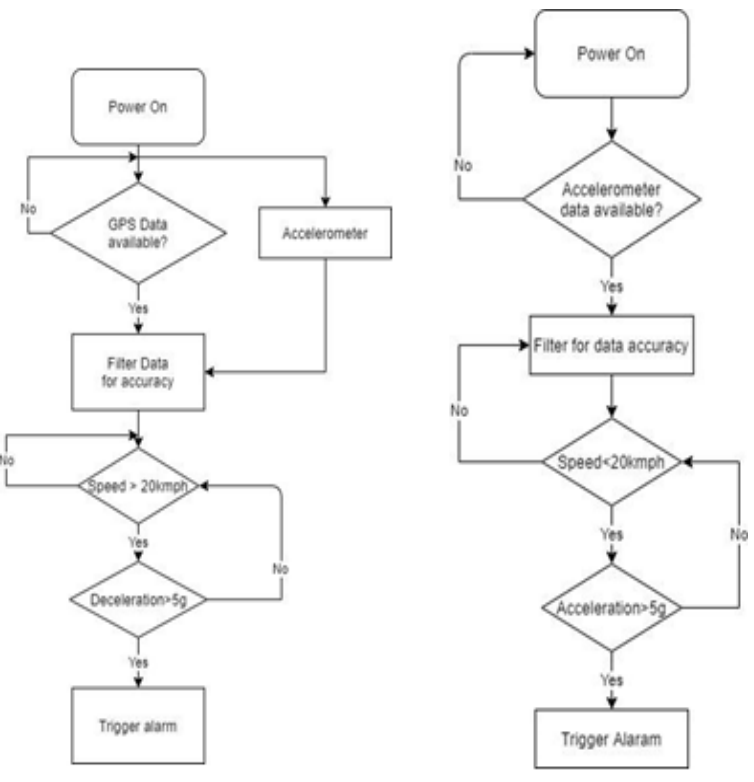

\subsection{Alarm Message and Location Detection:}

Once the accident is detected, the app displays a message on screen, asking if the user is injured. It also sets off a timer of 10 seconds. If the user does not respond to the message within this time, then the user is assumed to be badly injured and the call is made. If the user does respond, however, the activity is aborted.

\subsection{Calling the Ambulance:}

Once the location of the accident is determined, a message is generated specifying the location of the accident and the number of people involved in the accident. This message is converted to .mp3 form using android's TTS (text to speech API). Now the app makes a call to the designated 3 digit emergency number and plays the .mp3, letting the dispatcher know about the location of the accident.

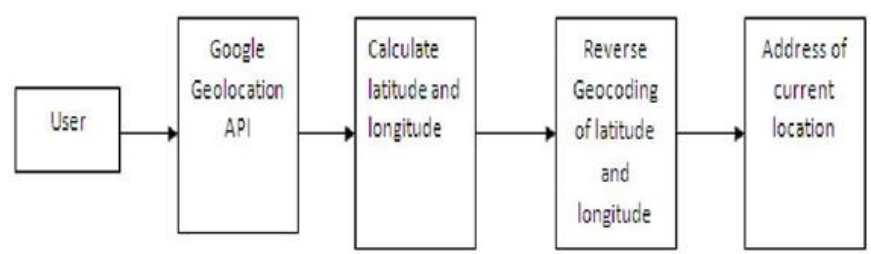

In 0 , sequence diagram shows the sequence in which emergency victims' application, Firebase and responders' application are performing their work. 
Sequence Diagram for Ambulance

\section{Sequence Diagrams}

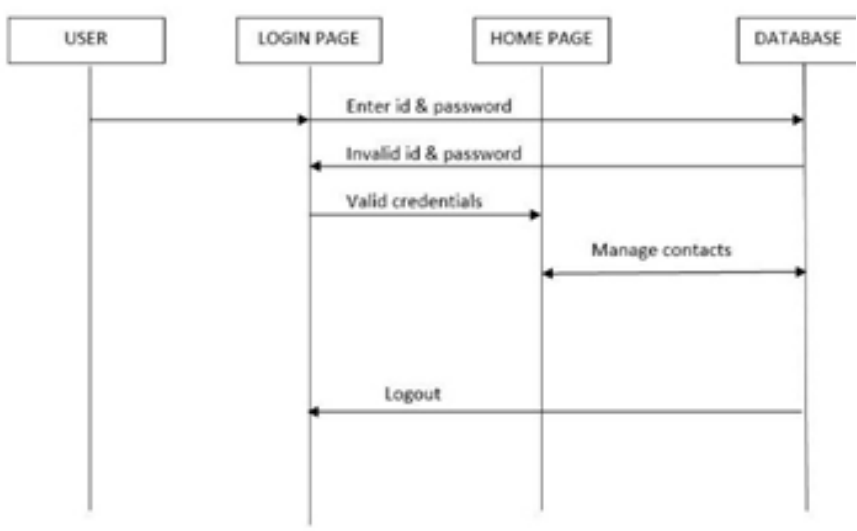

Sequence Diagram User

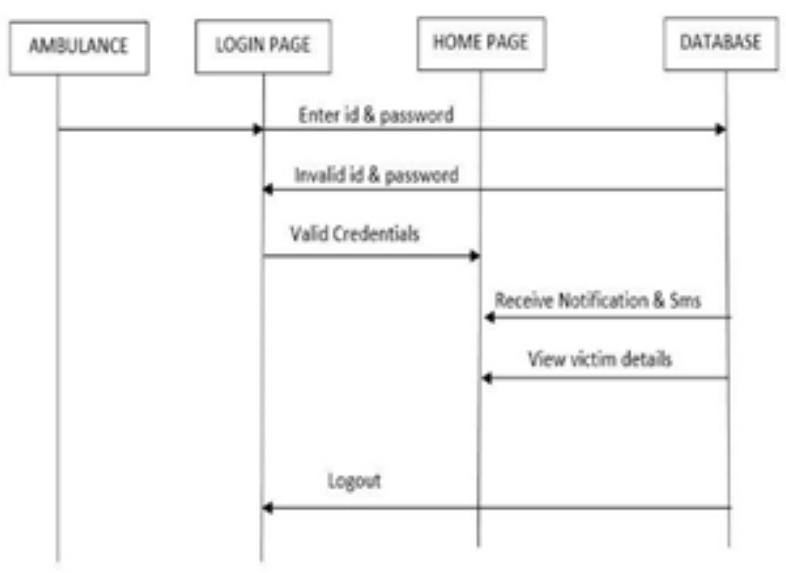

Sequence Diagram Ambulance

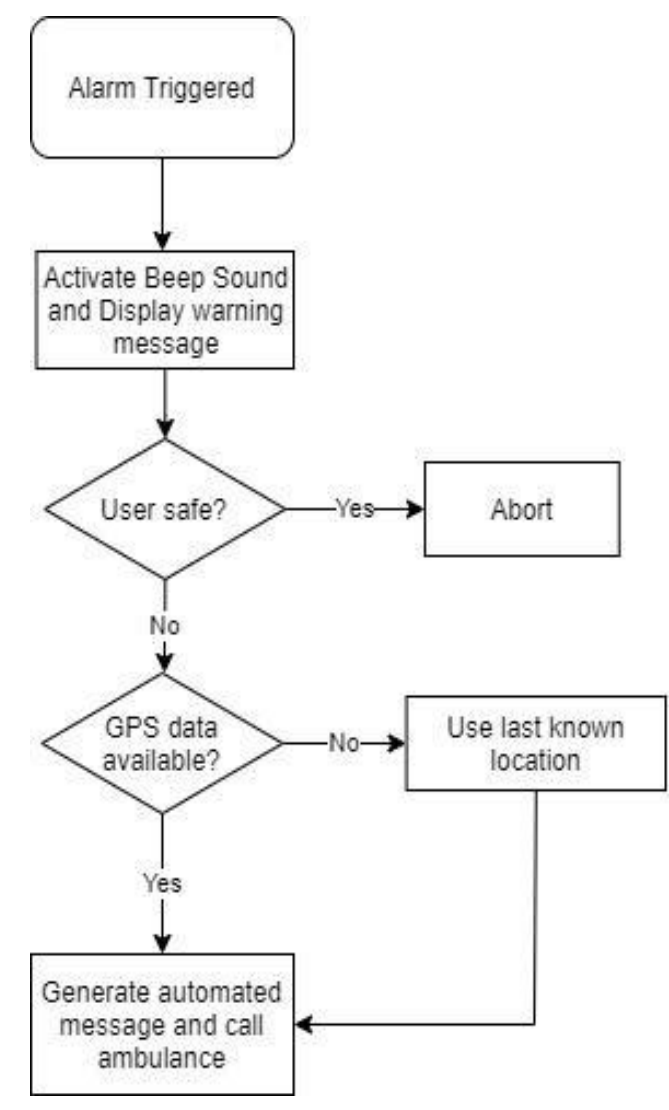

\section{CONCLUSION AND FUTURE WORK}

This research, we developed the accident detection and smart rescue system, which uses on board accelerometer sensor to detect accident and generate emergency alert and send it to the nearest emergency responder and will also send an SMS to emergency contact containing location coordinates of the accident. With real time location tracking for both victim and responder the system will drastically increase the survival rate of an accident victim by providing emergency aid in time. The system will also provide help during other emergencies such as during fire, robberies/theft and other medical emergencies. Emergency responder will be able pin point victim's location on a Google map in real time. The probability of false positives in a Smartphonebased accident detection and rescue system is inevitable. We have added some features to reduce these issues. Here are some features we added to reduce false positives. 
Acceleration filter: The system will ignore g-force values lower than $4 \mathrm{~g}$.

Count down timer alert: On detection of an accident the system will present an alert dialog with $15 \mathrm{sec}$ countdown, which the user will be able to cancel in case accident didn't occur.

For future work, more research is needed in order to make the accident detection part more reliable and accurate which will help in reducing false positives. Adding additional sensors in combination with accelerometer for accident detection like gyroscope, microphone, camera (to automatically take pictures of the accident) and a voice recognition module to detect noises during a vehicle crash like noise when air bags are deployed, will drastically increase the reliability and accuracy of the system.

\section{REFERENCES}

[1]. Manuel Fogue, PiedadGarrido, Francisco J. Martinez "A System for Automatic Notification and Severity Estimation of Automotive Accidents" IEEE January 2013

[2]. Tang Shumin et al., "Traffic Incident Detection Algorithm based on non-parameter regression"IEEE 5th International Conference on
Intelligent Transportation Systems, pp.714719,2002 EdmundasKazimierasZavadskas, RoodeLiias, ZenonasTurskis "MultiAttribute Decision Making Mathods of quality in bridges and road construction: State of Art Surveys", The Baltic journal of Road and Bridge Engineering, 2008

[3]. Deepak Punatha, Deepak Kumar, Vartika Mehta "Design and Realization of the Accelerometer based Transportation System"International Journal of Computer Applications (0975 - 8887) Volume 49- No.15, July 2012

[4]. William J. Jameson, Jr., MurariKejarawal, Troy Herreid, Charles Mitten and John M. Ray

\section{Cite this article as :}

Suhaib Ahmed, Srinidhi N, Sandhya Kyamma, Mohammed Imran, "Accident Detection and Safety System - (AUTO AID APP)", International Journal of Scientific Research in Computer Science, Engineering and Information Technology (IJSRCSEIT), ISSN : 2456-3307, Volume 7 Issue 4, pp. 62-66, July-August 2021. Available at doi : https://doi.org/10.32628/CSEIT217413 Journal URL : https://ijsrcseit.com/CSEIT217413 